\title{
Evaluation des risques sanitaires liés à l'alimentation de rue dans le District de Bamako
}

\author{
Risk Assessment related to Street Food in the District of Bamako \\ SAKO M1, KONE S1, YARO F1, TRAORE A1 DIALLO A1, SANGARE S1, DIAKITE F1 DIALLO F2, \\ KANOUTE G2 , DIARRA 01 ,CAMARA M1 CISSE D1 KEITA S1 .
}

1. Agence Nationale de la Sécurité Sanitaire des Aliments (ANSSA;)

2. Institut Supérieur de Formation et de la Recherche Appliquée (ISFRA)

Nous remercions l'ANSSA pour le financement de l'étude et l'ISFRA pour sa participation au travaux de recherche

\section{RESUME}

Au Mali, l'alimentation de rue s'est fortement développée ces dernières années avec l'effet de l'exode rural, la croissance démographique dans les villes et surtout avec l'instauration par l'Etat de la journée continue intervenue à partir de 1992 qui maintient les travailleurs hors de leur foyer pour de très longues heures.

La présente étude avait pour objectif d'évaluer les risques sanitaires liés à l'alimentation de rue dans le district de Bamako pour promouvoir la santé des consommateurs.

L'étude a porté sur un échantillon de 138 établissements dans les 6 communes du District de Bamako. II a été procédé à la détermination de la qualité sanitaire (microbiologique et chimique) des aliments de rue vendus dans le District de Bamako et à la classification des risques associés à l'alimentation de rue dans le district de Bamako, sous l'optique de la démarche du HACCP et de l'approche intégrée (vision FAO/OMS). Afin d'identifier les points critiques et de proposer des mesures de maitrise de la contamination microbienne 21 diagrammes de fabrication ont été élaborés et ont concerné tous les aliments contaminés. La classification des risques a été faite selon la procédure du Food and Drogue Administration des USA., outil internationalement reconnu et appliqué par le Codex Alimentatrus.

Sur les 59 échantillons analysés, 31 sont contaminés sur le plan bactériologique. Sur les 24 échantillons soumis à l'analyse toxicologique, 8 présentent des niveaux de résidus de pesticides élevés. Les résultats montrent (i) la majorité des établissements de production/vente d'aliments de rue qui n'applique pas les mesures d'hygiène et de fabrication (BPH/BPF) appropriées et (ii) un niveau de contamination élevé (46 - 62 sur l'échelle Risk Ranking du FDA) qui fait de l'alimentation de rue dans le District de Bamako une préoccupation en matière de sécurité sanitaire des aliments.

L'application des mesures correctives par le personnel des gargotes, a permis de maîtriser la contamination, ce qui est la preuve de la pertinence et l'efficacité de ces mesures.

Mots clés : aliments de rue, risques sanitaires, Bamako.

\section{SUMMARY}

In Mali, street feeding has food strongly expanded in recent years with the effect of the rural exodus, population growth in the cities and especially with the establishment by the State of the day occurred from 1992 that keeps workers out of their homes for very long hours.

This study was designed to assess the health risks related to Street food in the district of Bamako to promote the health of consumers.

The study focused on a sample of 138 facilities identified 170 in the 6 municipalities of the District of Bamako. It was made in the determination of the health quality (chemical and microbiological) of street foods sold in the District of Bamako and the classification of risks associated with food Street in the district of Bamako, the perspective of the process of HACCP and the integrated approach (vision $\mathrm{FAO} /$ who). To identify the critical points and to propose measures of control of microbial contamination, 21 manufacturing diagrams have been developed and have involved all contaminated food. The risk classification was made according to the procedure of the FDA of the USA.

On the 59 samples, 31 were contaminated on the bacteriological plan. The 24 samples subjected to toxicological analysis, 8 have high pesticide residue levels.

The results showed (i) a high rate of establishment of production/sale of street food that does not appropriate measures of hygiene and manufacturing (BPH/GMP) and (ii) a level of high contamination (46-62 on the Risk Ranking of the FDA scale) makes food Street in the District of Bamako a food safety concern.

The application of corrective measures by the staff of the eateries, allowed to control contamination, which is evidence of the relevance and effectiveness of these measures.

Keyword (s): street food, health risks, Bamako.

\section{INTRODUCTION}

Les aliments de rue représentent une part importante de la consommation alimentaire urbaine journalière pour les populations à revenus faibles ou moyens qui représentent la grande majorité des citadins. Cette alimentation concerne principalement les pays en développement [FAO, 2007].

La FAO et l'OMS ont entrepris dès les années 1980 des actions de renforcement de l'hygiène des aliments produits et vendus dans les rues, en privilégiant une 
approche intégrée rassemblant des différents partenaires tels que les vendeurs, les préparateurs, les consommateurs, les autorités municipales, les services techniques en charge des contrôles et les institutions de recherche et de développement [FAO, 2007].

Le risque d'intoxication alimentaire est une menace pour tous les pays du monde mais singulièrement pour ceux en développement dont le Mali. II est reconnu que les agents pathogènes d'origine alimentaire représentent un grave danger pour la santé publique.

Le risque dépend aussi bien de l'agent pathogène que du type d'aliment, de la méthode de préparation et de conservation de l'aliment. L'ignorance des vendeurs des aliments de rue est aussi un facteur qui aggrave les risques. Le manque d'hygiène, la non disponibilité d'eau potable, l'insalubrité des lieux de vente, la promiscuité avec les dépôts d'immondices ou les égouts d'évacuation des déchets, sont autant de préoccupations pour les services de l'Etat et les organisations de la société civile en charge de la protection de la santé des populations [FAO, 2007, Outils pour la formation]. L'emploi irraisonné des additifs ou des compléments alimentaires, des colorants pour rendre ces aliments destinés au grand public plus attrayants, ajouté aux résidus de produits vétérinaires ou phytosanitaires et à l'utilisation de papier d'emballage inapproprié pour ne citer que cela ; font de l'alimentation de rue un cocktail explosif à surveiller de très près. [FAO, 2007].

En milieu informel où on ne procède à aucune analyse des aliments, à aucun enregistrement, le système HACCP peut être appliqué dans les établissements de préparation d'aliments de rue en se basant sur les observations visuelles des produits, des tests simples de contrôle de la température, de l'acidité etc. Ces actions tout en améliorant la qualité sanitaire des aliments, n'engendreront pas de charges excessives.

La présente étude a pour objet d'utiliser la démarche HACCP, système de prévention et d'anticipation des risques sanitaires pour donner des orientations aux opérateurs impliqués dans l'alimentation de rue, faire des prescriptions techniques relatives aux dangers que peuvent véhiculer les produits finis à l'attention des services de surveillance relevant des collectivités territoriales, qui sont très impliquées dans l'hygiène et la salubrité des aliments. Avec l'application de ces orientations, les gargotes pourraient se définir comme étant des restaurants où l'on mange à bas prix une nourriture saine.

\section{MATERIEL ET METHODE}

L'étude a été descriptive, analytique et évaluative basée sur la méthode de l'évaluation des risques du Codex Alimentarius et du Food and Drogue Administration (FDA).

La Méthode d'échantillonnage a été probabiliste pour l'état des lieux des établissements et raisonnée pour les phases HACCP à partir des 32 critères de la grille de Beter Training for Safer Food. Le Choix a été exhaustif pour les aliments contaminés et la commodité pour les clients
Le CADRE de l'étude a été les 6 communes du district de Bamako et les populations concernées par l'étude ont été les gérants /vendeurs d'aliments de rue à des points fixes comme cibles primaires et les clients des gargotes comme cibles secondaires.

Tous les aliments qui étaient prêts au passage de l'équipe d'enquête ont constitué 144 échantillons d'aliment cuisinés, crudités et eau plate ont été prélevés par les technicien de laboratoires (Laboratoire Central Vétérinaire et Laboratoire National de la Santé ). Les échantillons prélevés ont été envoyés pour analyse au Laboratoire Central Vétérinaire pour la recherche des résidus de pesticides ou au Laboratoire National de la Santé pour la recherche microbiologique.

Les données ont été collectées à l'aide de questionnaire et de l'observation des procédés.

Le questionnaire à l'endroit des opérateurs comportait les éléments suivants les renseignements généraux, la Chaîne alimentaire, l'approvisionnement en eau, la gestion des déchets, l'hygiène du personnel, l'existence de méthodes d'assurance qualité. Le questionnaire à l'endroit des Clients avait retenu les renseignements généraux, la gestion des cas de maladies d'origine alimentaire, les suggestions et recommandations.

La grille d'observation du procédé contenait comme indications : l'environnement de production, la Chaîne alimentaire, les Matières premières et ingrédients, matériel et aliments, l'approvisionnement en eau, la gestion des déchets, l'hygiène du personnel et l'existence de méthodes d'assurance qualité.

Les données ont été analysées et traitées avec le logiciel EPI INFO, version 6.04. Les résultats et les caractéristiques générales sont présentés sous forme de tableaux ou de figures. L'analyse a comporté deux phases : une phase descriptive et une phase analytique.

\section{RESULTATS}

Les risques liés à l'alimentation de rue dans le district de Bamako ont été évalués ; des mesures pour leur maîtrise dans le but de promouvoir la santé des consommateurs, ont été précisées.

Afin d'aboutir à cet objectif, des dispositions ont été mises en œuvre pour une approche méthodique à partir des fiches d'enquêtes. A cet effet, 27, 5\% des établissements enquêtés avaient une source potentielle de contamination dans un rayon de moins de $5 \mathrm{~m}$. Les caniveaux constituaient $86,8 \%$ des sources potentielles de contamination. Les établissements avec toilettes ne representaient que $18,8 \%$ et $(23,1 \%$ des toilettes étaient situées à moins de $10 \mathrm{~m}$ de la cuisine. La disponibilité de poubelles dans les établissements enquêtés était de $95,7 \%$ avec $80,3 \%$ qui étaient à ciel ouvert. Près de $42,8 \%$ des établissements conservaient les matières premières à la température ambiante.

Près de $71 \%$ des établissements nettyaient et désinfectaient les crudités dont $69,4 \%$ avec eau de Javel. La réparation des aliments se faisait en plein air par $32,6 \%$ des établissements. La quasi-totalité des opérateurs $(99,3 \%)$ n'avaient pas été formé aux bonnes pratiques d'hgiène. 
Le suivi des gargotes par les services techniques de l'état était de $13,8 \%$ au plan médical, de $52,9 \%$, en terme d'hygiène et par la mairie dans $39,7 \%$.

Aucune démarche qualité n'avait été observée dans les gargotes étudiées

\section{Aliments à risque :}

Les moyennes de contamination réelle des aliments étaient de :

Contamination du riz avec sauce tomate par les coliformes thermo tolérants : 2/6 échantillons avec une moyenne de 3,62 germes; les anaérobies sulfito réducteurs: 2/6 échantillons avec une moyenne de 3,14 germes; les levures et moisissures : 1/6 échantillons avec une contamination moyenne de 2,60 germes, les coliformes thermo tolérants : $2 / 2$ échantillons avec une moyenne de 2,82 germes

Contamination du riz avec sauce arachide par : les anaérobies sulfito - réducteurs: $1 / 2$ échantillons avec une contamination de 2,60 germes ; les streptocoques fécaux: $1 / 2$ positif.

Contamination du riz au gras par les coliformes thermo tolérants: $1 / 3$ échantillons avec une contamination de 4,07 germes, les levures et moisissures: $1 / 3$ avec une contamination de 2,60 germes.

Contamination du riz avec sauce yassa par les streptocoques fécaux : positif $1 / 1$

Contamination du Sandwich par les anaérobies sulfito - réducteurs: $1 / 2$ échantillons avec une contamination de 3,04 germes

Contamination du poulet par les coliformes thermo tolérants : 1/1 échantillon avec une contamination de 3,07 germes

Contamination du riz décortiqué par les levures et moisissures: 6/15 échantillons avec une moyenne de 2,19 germes

Contamination du fonio précuit par les levures et moisissures: $2 / 3$ échantillons avec une moyenne de 3,15 germes.

Contamination du mil par les levures et moisissures: 1/1 échantillon avec une moyenne de 2,60 germes

Contamination de la farine de maïs par les levures et moisissures: 1/1échantillon avec une moyenne de 2,00 germes

Contamination de bissap par les streptocoques fécaux : positif $1 / 1$

Contamination des crudités par les Coliformes thermo tolérants : $3 / 6$ avec une moyenne de 3,03 germes.

\section{Recherche de résidus de pesticides.}

Sur les 6 échantillons de salade analysés, tous contenaient des résidus de cyhalotrine avec une moyenne de 2,53.

Riz décortiqué :

Sur 15 échantillons de riz analysés, 2 contenaient des résidus de cyhalotrine avec une moyenne de 1,46.

Le fonio, le mil et la poudre de mais ne contenaient pas de résidus de pesticides.

\section{DISCUSSIONS}

Nos résultats ont montré l'efficacité des mesures correctives. La vérification (analyse de tous les aliments contaminés de l'état des lieux) a prouvé la pertinence et l'efficacité des mesures. Tous les échantillons prélevés pour vérification( application des mesures correctives d'application des bonnes pratiques d'hygiène et de fabrication ) se sont révélés conformes aux normes sanitaires.

La restauration sociale, "rôtisseries/dibiteries", vente de brochettes, aliments ambulants non pas été pris en compte dans l'étude. Le nombre de gargotes pour les plans HACCP était faible; 22 plans HACCP prévus, 19 réalisés pour 15 gargotes. Pour les analyses bactériologiques les choix ont porté sur E.coli pour les Coliformes Thermo Tolérant (CTT) et Clotrisduim botulinum pour Anaérobie Sulfito R.éducteurs(ASR).

Les hypothèses ont été confirmées quand au risque chimique lié à l'alimentation de rue Bamako révélé I par les analyses toxicologique (résidus de $\lambda$ cyhalothrine entainant l'irritation des voies respiratoires, nocif au contact peau ou avalé) et la possibilé de formation de dioxyne et, acrylamine par le mode préparation des aliments. Seulement $42,0 \%$ des clients ont exigé le respect des mesures d'hygiène. Le risque microbiologique a été circonscrit par les mesures correctives proposées par l'étude.

"La valeur de " Risk Ranking" est une mesure simplifiée du risque. En raison de l'ampleur des différences dans les risques eu égard aux situations et aux scénarios d'intérêt des questions de santé publique, une échelle logarithmique est utilisée et pour plus de commodité, une échelle entre 0 et 100 a été choisie. La limite supérieure de l'échelle était fixée à (100)/ Elle a représenté le pire scénario imaginable, c'est à dire le cas où chaque membre de la population consommait un repas qui contenait une dose mortelle du danger et cela tous les jours. Selon la classification qui a été faite ; les notes ont été de 46 à 62 sur une échelle de 0 à 100. Cependant, des études supplémentaires doivent être faites pour préciser l'implication des pathogènes dans les toxi-infections alimentaires collectives (TIAC) afin de pouvoir déterminer les doses infectantes et létales dans les conditions du pays. Les résultats des analyses de laboratoire n'vaient pas précisé l'espèce de la famille du pathogène. L'exercice de classification a été fait en ciblant les espèces les plus fréquentes ou les espèces les plus pathogènes dans les aliments. II s'agssait d'Escherichia coli pour les Coliformes thermo tolérants et du Clostridium botulinum pour les anaérobies sulfito réducteurs.

\section{CONCLUSION}

Nos résultats montraient que les niveaux de risques étaient élevés (46 - 62 sur l'échelle 100 du Risk Ranking). Cela fait de la qualité sanitaire des aliments de rue une préoccupation majeure des autorités nationales chargées de la protection de la santé des consommateurs. Les mesures correctives développées, qui ont prouvé leur pertinence et leur efficacité, sont 
faciles à mettre en œuvre et n'exigent aucune dotation complémentaire ni en personnel ni en équipement. Leur vulgarisation concourerait sans doute à l'amélioration de la qualité sanitaire des aliments et par conséquent à la protection de la santé des consommateurs d'aliments de rue.

L'étude s'est limitée aux dangers microbiologiques. Les dangers physiques sont facilement discernables et facilement éliminés par des dispositions de salubrité (vannage, lavage, le tri par exemple pour les corps étrangers). Quant aux dangers chimiques, ils mériteraient d'être évalué dans les aliments de rue.

\section{REFERENCES}

1. FAO.

"Les bonnes pratiques d'hygiène dans la préparation et la vente des aliments de rue en Afrique : outils pour la formation, (2007).

2. ANSSA.

Rapport final étude viande rouge, 2007.

3. KONE Salimata,

Evaluation de l'efficacité de la prise en charge du diabète de type 2 et les facteurs associés dans la ville de Cotonou, mémoire de master en santé publique. IRSP, 2005.
4. DIATTA J.

Bureau de Contrôle des Produits Halieutiques/Direction des Industries de Transformation de la Pêche Sénégal : Le Concept HACCP : Application dans les marchés au poisson.

5. NIAGALY. Adégne.

Etude des toxi-infections alimentaires collectives en république du Mali, 2006,

6. Mairie Centrale du District de Bamako Site officiel de la ville de Bamako.

\section{FAO/OMS}

Directives pour le renforcement des systèmes nationaux de contrôle alimentaire,

8. PRODESS II.

Composante santé Programme de Développement Socio Sanitaire 2005 - 2009, (2004),

9. Kendall M.G, Smith B.B.

Table of Random Numbers », " Randomness and Random Sampling Numbers, « Journal of the Royal Statistical Society, 1938, vol 101.

10. Laboratoire d'hygiène Lyonnais. Les différents germes. 